\title{
Deutsche wirtschaftspolitische Reformberatung in China am Beispiel der GTZ
}

\author{
André Lieber
}

\begin{abstract}
Kernaussagen
Nachdem im Juli 2010 die klassische bilaterale Entwicklungszusammenarbeit formal beendet wurde, kam es im Sommer $2011 \mathrm{zu}$ den ersten deutsch-chinesischen Regierungskonsultationen. Am Beispiel der wirtschaftspolitischen Reformberatung der deutschen Gesellschaft für Technische Zusammenarbeit (GTZ) $\mathrm{GmbH}^{1}$ - und hierbei speziell dem Programm Wirtschafts- und Strukturreform und seiner institutionellen Vorläufer - liefert der vorliegende Bericht eine kleine Historie deutscher wirtschaftspolitischer Reformberatung in China. Als Erfolgsfaktoren der Zusammenarbeit können dabei unter anderem die personelle Kontinuität, eine sorgfältige Auswahl der Kooperationspartner und die Einbeziehung internationaler Perspektiven gelten.
\end{abstract}

\section{Die Phasen der chinesischen Wirtschaftsreform seit Ende der 1970er Jahre}

In vielen aktuellen wirtschaftspolitischen Debatten, von der Staatsschuldenkrise über die Förderung erneuerbarer Energien bis hin zur Zukunft des Euros, kommt früher oder später die Rede auf China und seine Wirtschaftsmacht. Dabei bewegt sich die deutsch-chinesische Zusammenarbeit insgesamt in einem Spannungsfeld aus wachsender gegenseitiger Neugier und einem andauernden Prozess institutioneller Veränderung.

Rückblickend lassen sich drei zentrale Phasen in der chinesischen Wirtschaftsreform seit Ende der 1970er Jahre abgrenzen . Nach der schrittweisen Machtübernahme durch eine Gruppe der Wirtschaftsreformer um Deng Xiaoping war die erste Phase (1978-1989) durch eine bewusste Legitimierung des Marktes und marktwirtschaftlicher Lösungen gekennzeichnet. Plan- und marktwirtschaftliche Elemente existierten vielfach nebeneinander und das Augenmerk galt vor allem einer Reform der ökonomischen Anreizstrukturen. Nach einer Übergangsphase schloss sich eine zweite Reformphase (1993-2001) an, in der es vor allem um die Legitimierung des Staates und staatlicher Eingriffe ging. Folgerichtig rückten fiskal- und finanzpolitische Aspekte und die Leistungsfähigkeit öffentlicher Verwaltung ins Zentrum der Aufmerksamkeit. Nach einer weiteren Übergangsphase befindet sich die chinesische Wirtschaft nun in einer dritten Reformphase (2005-heute), in der es vorrangig um die Legitimierung der Gesellschaft und das reibungslose Zusammenspiel zwischen Markt, Staat und Bürgern geht. Wichtige Stichworte sind der Aufbau einer harmonischen Gesellschaft und die allgegenwärtige Forderung nach einer bürgerlichen Gesinnungs- und Handlungsethik beziehungsweise effizienter sozialer Kontrolle.

\section{Deutsche wirtschaftspolitische Reformberatung in China}

\subsection{Erste Reformphase: Legitimierung des Marktes (1978-1989)}

Nach Chinas erfolgreichem Beitritt zur Weltbank und zum Internationalen Währungsfonds unterzeichneten eine Reihe von Staaten bilaterale Kooperationsverträge - so 1982 auch Deutschland. Im Auftrag des damaligen Bundesministeriums für Wirtschaftliche Zusammenarbeit engagierte sich die GTZ in den kommenden Jahren vor allem in den Bereichen Armutsbekämpfung und berufliche Bildung. Alle Projekte wurden von der Eschborner Zentrale aus gesteuert, eine unter internationalen Geberorganisationen damals weit verbreitete Vorgehensweise. Die deutsche Seite sorgte in aller Regel für die materielle Grundausstattung der Projekte und leistete Hilfestellung bei der Aus- und Weiterbildung von Fachkräften.

Vor dem Hintergrund der von der Kommunistischen Partei Chinas (KPC) seit 1984 proklamierten 'sozialistischen planwirtschaftlich-orientierten Warenwirtschaft' einigten sich die deutsche Botschaft und das chinesische Handelsministerium zur Jahreswende 1986/87 auf ein gemeinsames Austauschprogramm für Nachwuchskräfte der staatlichen Planungskommission (State Planning Commission, SPC). Im Rahmen des so genannten NPTC-Projekts (National Planers“ Training Center) wurden vier Teilprojekte umgesetzt:

1. Vortragsreisen hochrangiger deutscher Wirtschaftsexperten: Experten wie beispielsweise Prof. Willy Kraus und Prof. Hans Schneider (ehemals Vorsitzender des Sachverständigenrats) führten zahlreiche Gespräche mit chinesischen Wirtschaftspolitikern und referierten über die Prinzipien deutscher Wirtschaftspolitik. Im Sommer 1987 kam es u.a. zu einem Treffen zwischen Prof. Schneider und dem damaligen Ministerpräsidenten Zhao Ziyang. Das Teilprojekt wurde nach den Protesten im Sommer 1989 zunächst unterbrochen.

2. Einmonatige Chinaaufenthalte hochrangiger deutscher Beamter finanziert durch die Deutsche Stiftung für inter-

1 Zum 1. Januar 2011 wurden der Deutsche Entwicklungsdienstes (DED), die Deutsche Gesellschaft für Technische Zusammenarbeit (GTZ) $\mathrm{GmbH}$ und die Internationale Weiterbildung und Entwicklung gGmbH (InWEnt) unter dem Dach der neu gegründeten Deutschen Gesellschaft für Internationale Zusammenarbeit (GIZ) fusioniert. Der Bericht beruht zum Großteil auf persönlichen Interviews mit GIZ-Mitarbeitern und chinesischen Projektpartnern, die im April/Mai 2011 in Deutschland und China durchgeführt wurden. 
nationale Entwicklung (insgesamt 35 deutsche Teilnehmer).

3. Einjährige Deutschlandaufenthalte für junge Nachwuchskräfte der staatlichen Planungskommission: Zwischen 1988 und 1995 haben 70 chinesische Nachwuchskräfte an diesem Stipendiatenprogramm teilgenommen und neben Sprachkursen auch mehrmonatige Praktika in deutschen Unternehmen absolviert.

4. Ausstattung chinesischer Institutionen mit Büromaterialien und wissenschaftlichen Publikationen.

Der Kontakt zwischen dem chinesischen Alumni-Netzwerk, den deutschen Beratern und einflussreichen Zirkeln chinesischer Wirtschaftspolitiker war von Anfang an sehr eng. Ein Beispiel: Im Juli 2000 wechselte ein Teilnehmer des Stipendiatenprogramms zur GTZ. Dieser Mitarbeiter war während seines Ökonomiestudiums an der Universität Peking im persönlichen Think Tank von Zhao Ziyang tätig, dem Chinese Economic System Reform Research Institute (SRI). Das SRI, gegründet im Jahr 1985, brachte vor allem junge Wirtschaftsreformer zusammen; musste allerdings im Sommer des Jahres 1989 schließen beziehungsweise wurde mit einem konservativen Think Tank zwangsfusioniert. Zu den bekanntesten und einflussreichsten Forschern des SRI zählen u.a. Lu Mai (heute: China Development Research Foundation) und Zhou Qiren (heute: China Centre for Economic Research, CCER) .

Im Laufe der Jahre verlagerte sich der Beratungsschwerpunkt der GTZ zunehmend von der mikro- auf die makropolitische Ebene. Zum Jahreswechsel 1989/1990 wurde darüber hinaus das Pekinger GTZ-Verbindungsbüro eröffnet.

\subsection{Zweite Reformphase: Legitimierung des Staates (1993-2001)}

Auch die deutsche Beratung erhielt mit Deng Xiaopings Reise in den Süden neuen Schwung. Unter dem neuen Leitbild der 'sozialistischen Marktwirtschaft' ging es in den 1990er Jahren um konkrete Transformationsberatung sowie den Aufbau marktwirtschaftlicher Institutionen. Zum Projekt mit der Planungskommission kamen neue Partnerschaften mit dem China Institut für Reform und Entwicklung (CIRD) und der Strukturreformkommission (State Council Office for Restructuring the Economic System, SCORES) hinzu. Bei der Beratung zur Reform der Staatsbetriebe konnte die GTZ gezielt auf deutsche Erfahrungen im Zuge der Wiedervereinigung zurückgreifen (Treuhandanstalt, Bundesfinanzministerium, Bundeswirtschaftsministerium). Darüber hinaus wurde über das Centrum für internationale Migration und Entwicklung ein deutscher Langzeitexperte für Restrukturierungsfragen in den liberalen Think Tank Unirule entsandt .

Der Leiter von CIRD, Prof. Chi Fulin, ehemals Mitarbeiter im Büro für politische Reformen von Ministerpräsident Zhao Ziyang, hatte sich nach den Protesten im Jahr 1989 ganz bewusst dem Einfluss Peking entzogen und war mit seinem neugegründeten Institut in den Süden Chinas umgesiedelt . Der Süden und speziell die Insel Hainan boten sich als Aus- weichquartier an, denn hier konnten Reformthemen, die in Peking noch als zu sensibel eingestuft wurden, ohne größere Probleme offen angesprochen und diskutiert werden. Die Zusammenarbeit mit CIRD erhielt darüber hinaus einen erneuten Schub mit der Ankunft eines deutschen Langzeitexperten im Juli 1998. Im gleichen Jahr erweiterte das Institut sein Themenspektrum um Fragen des internationalen Handels. In enger Abstimmung mit dem in Genf ansässigen International Trade Center und dem WTO-Sekretariat wurde ein Dokumentationszentrum eingerichtet, das später als offizielles WTO-Referenzzentrum anerkannt wurde. Darüber hinaus erhielt die GTZ im Mai 2000 den Zuschlag für die erste Phase des EU-Projekts zum WTO-Beitritt Chinas.

Ein weiterer wichtiger Bestandteil der deutschen Reformberatung war zu dieser Zeit ein Ausbildungsprogramm für chinesische Manager mittelständischer Unternehmen. Die Teilnehmer durchliefen ein Trainingsprogramm und hospitierten in deutschen Betrieben. Im Jahr 2000 wurde außerdem zusammen mit der staatlichen Kommission für Wirtschaft und Handel (State Economic and Trade Commission, SETC) und weiteren deutschen Partnern (SEQUA, AHK Peking) ein Beratungsprojekt für kleine- und mittelständische Unternehmen ins Leben gerufen. In zwei Pilotprovinzen wurden lokale Behörden bei der Privatsektorentwicklung und dem Aufbau von Kammern und Verbänden unterstützt.

Auf Einladung der GTZ, der deutschen Botschaft und des Ministeriums für Außenhandel und wirtschaftliche Zusammenarbeit (MOFTEC) fand im September 2000 in Peking ein Planungstreffen statt. Ziel des Treffens war die Konsolidierung und strategische Neuausrichtung der deutschen wirtschaftspolitischen Beratung in China.

\subsection{Dritte Reformphase: Legitimierung der Gesellschaft (2002-heute)}

Der Jahreswechsel 2002/03 markierte auch für die GTZ China eine Übergangsphase. Zwar gab es noch einzelne Beratungsprojekte und die Beteiligung am EU-WTO-Projekt, doch noch fehlte eine inhaltliche und organisatorische Klammer. Ab dem Jahr 2003 begann sich die gesamte GTZ China auf vier Schlüsselthemen zu konzentrieren: Wirtschaftsreform und Aufbau der Marktwirtschaft (WIRAM), Ressourcenschutz und Armutsbekämpfung, Umweltschutz und Energiemanagement.

Im April 2004 wurden dann zum ersten Mal alle Vorhaben der deutschen Seite in einem einheitlichen Programm Wirtschafts- und Strukturreform zusammengefasst. Dieser Schritt erlaubte nicht nur eine bessere Vernetzung der chinesischen Partner, sondern auch mehr tagespolitische Flexibilität. Neben der Weiterentwicklung der 'sozialistischen Marktwirtschaft' ging es den Partnern in dieser Phase vor allem um einen fachlichen Austausch zur Ausgestaltung und praktischen Umsetzung wirtschaftspolitischer Leitbilder wie der Gesellschaft mit bescheidenem Wohlstand (xiaokang shehui), der harmonischen Gesellschaft (hexie shehui) und dem wissenschaftlichen Entwicklungskonzept (kexue fazhan guan). Darüber hinaus waren einige der Partner sehr eng in die laufenden Vorberei- 
tungen des 11. Fünf-Jahres-Plans (2006-2010) eingebunden. Die erste Programmphase (April 2004 bis Oktober 2007) beinhaltete zunächst drei Komponenten:

1. Zusammenarbeit mit der Nationalen Kommission für Entwicklung und Reform (NDRC) (volkswirtschaftliche Prognosen, Modellierung von Gleichgewichtsmodellen, regionale Entwicklungsstrategien)

2. Zusammenarbeit mit dem Forschungsbüro des Staatsrats (State Research Office, SRO) im Bereich Wirtschafts- und Sozialpolitik (industriepolitische Cluster, Strukturwandel, Strategie zur Revitalisierung des Nordostens, Wanderarbeiter)

3. Zusammenarbeit mit CIRD und anderen Forschungsinstituten wie dem Development Research Center of the State Council (DRC) und der CASS zu Fragen der Wirtschafts-, Verwaltungs- und Sozialreform (Mikrokredite, soziale Marktwirtschaft, Sozialversicherungssysteme)

Die Art der Zusammenarbeit war naturgemäß von unterschiedlicher Intensität. Während NDRC als einer der einflussreichsten Akteure der chinesischen Politik großen Wert auf enge politische Abstimmung legt, möchte sich SRO als eine Art Agenda-Setter profilieren.

Ab dem Frühjahr 2005 kamen noch zwei weitere Komponenten zum Program hinzu:

1. Zusammenarbeit mit dem Büro der Führungsgruppe für Finanzen und Wirtschaft (Office of the Leading Group on Finance and Economic Affairs, OLGFE) zu Themen der regionalen Entwicklung

2. Zusammenarbeit mit dem China Training Center for Senior Personnel Management Officials (CTCSPMO oder kurz CTC) zu Themen der politischen Führung und der Weiterbildung von Parteikadern ${ }^{2}$

Die Arbeitskontakt mit der Führungsgruppe für Finanzen und Wirtschaft wiederum ist stark durch den bekannten Wirtschaftspolitiker Liu He geprägt, der in verschiedenen Medien auch schon als "Chinas Larry Summers" portraitiert wurde . Zusammen mit NDRC und der Führungsgruppe wurde ebenfalls im Jahr 2005 ein sehr hochrangiger regional- und industriepolitischer Dialog ins Leben gerufen, in den u.a. auch die OECD, die EU-Kommission und UNIDO mit eingebunden sind.

Im Rahmen des BMZ-Ankerlandkonzepts engagierte sich das Programm zudem in der internationalen entwicklungspolitischen Debatte. Die chinesischen Partnerinstitutionen wurden systematisch in die Arbeit mit regionalen Dialogforen wie beispielsweise der Economic Commission for Latin America and the Caribbean oder regionalen Initiativen der Asiatischen Entwicklungsbank wie der Greater Mekong Subregion Initiative oder dem Pan-Beibu Gulf Economic Cooperation Forum mit einbezogen.

Ende des Jahres 2007 wurde das Programm Wirtschaftsund Strukturreform dann neu aufgestellt. Die zweite Programmphase umfasste erneut fünf Komponenten: gesamtwirtschaftliche Politik und Strukturreform (NDRC), wirtschafts- und sozialpolitische Entscheidungsvorlagen für den Staatsrat (SRO), Forschung zur Wirtschafts-, Verwaltungs- und Sozialreform (CIRD und andere Forschungsinstitute), ${ }^{3}$ Entwicklung von Distributionssystemen im ländlichen Raum (MOFCOM), nachhaltige Regionalentwicklung (Büro der Führungsgruppe für Finanzen und Wirtschaft). Dazu kommt noch die weitergeführte Kooperation mit CTC zum Thema Verwaltungsreform und Qualifizierung von Parteikadern. Zu den Partnern dieses personalpolitischen Dialogs gehören u.a. das Bundesinnenministerium, die Bundesakademie für öffentliche Verwaltung und die Deutsche Hochschule für Verwaltungswissenschaften. Auf europäischer Ebene ist darüber hinaus das Europäische Institut für Öffentliche Verwaltung in Maastricht eingebunden.

Mit Ausnahme der vierten Komponente mit Aktivitäten in den Provinzen Hebei und Anhui ist das Programm aufgrund seiner chinesischen Partner in erster Linie auf die zentralstaatliche Ebene fokussiert. Allerdings werden immer wieder Erfahrungen aus den Provinzen beispielsweise in Form von Fallstudien und Forschungsberichten über erfolgreiche Pilotmaßnahmen in die tägliche Arbeit eingebracht. Durch die Nachwirkungen der internationalen Finanzmarktkrise waren alle chinesischen Partner zeitlich sehr stark belastet. Die Krise und ihre Bekämpfung eröffnete aber auch neue Themen; so kam es u.a. mit Prof. Peter Bofinger vom Sachverständigenrat zu einem Fachdialog über die Wirksamkeit von Konjunkturpaketen. Der Beratungsschwerpunkt lag weiterhin auf der Umsetzung des 11. Fünf-Jahres-Plans.

Ende 2011 wird diese zweite Programmphase auslaufen, wobei die Komponenten (1) und (5) zwischenzeitlich schon bis 2014 verlängert worden sind. D.h. es wird auch in Zukunft eine deutsche Reformberatung geben und auch der hochrangige Dialog mit der Führungsgruppe zum Thema nachhaltige Regionalpolitik wird ebenfalls fortgesetzt. Für diese dritte Programmphase stehen dann vor allem die Themen des 12. FünfJahres-Plans im Mittelpunkt:

- Transformation des Wachstums- und Entwicklungsmodells

- Weiterentwicklung zu einer energieeffizienten und emissionsarmen Gesellschaft

- Koordination einer nachhaltigen Regionalpolitik

- Maßnahmen zur sozialen Sicherung und Verbesserung des Zugangs zu öffentlichen Gütern und Dienstleistungen

\section{Fazit}

Stand 2011 ist das Programm Wirtschafts- und Strukturreform gut in innerchinesische Reformdebatten eingebettet, wobei sich das Leitbild der sozialen und ökologischen Marktwirtschaft als international anschlussfähig erwiesen hat.

2 Die Zusammenarbeit mit CTC begann als Gemeinschaftsproduktion von GTZ und InWEnt.

$3 \mathrm{Zu}$ den anderen Forschungsinstituten gehören u.a. die Chinesische Akademie für Sozialwissenschaften (CASS), die China Development Research Foundation, die China Foreign Affairs University (CFAU) und die Chinesischen Verwaltungsakademie (Chinese Academy of Governance, CAG). 
Gleichzeitig ist eine effektive und effiziente wirtschaftspolitische Beratung Dritter nur möglich, wenn man die politischen, sozialen und kulturellen Hintergründe des jeweiligen Partners kennt. Die GTZ/GIZ China und speziell das Programm Wirtschafts- und Strukturreform und seine zahlreichen institutionellen Vorläufer waren in der Vergangenheit sehr erfolgreich, sich diese Kenntnisse anzueignen und für deutsche Interessen nutzbar zu machen. Dieser Erfolg beruhte im Wesentlichen auf personeller Kontinuität, einer aufwendigen Auswahl der chinesischen Partner und der frühzeitigen Integration internationaler Perspektiven. Schließlich hat es das Programm vermocht, den chinesischen Reformprozess als einen „Prozess institutioneller Innovation" anzunehmen und seine Arbeitsweise entsprechend zu gestalten. Bis heute hat sich das Programm und sein Umfeld eine gewisse institutionelle Neugier bewahren können.

\section{Literatur}

Li, C. (2010). Shaping China's Foreign Policy: The Paradoxical Role of Foreign-Educated Returnees. Asia Policy, July(10), 65-85.

Naughton, B. (2009). Singularity and Replicability in China's Developmental Experience (preliminary version of a paper presented at the 2009 AEA Meetings, San Francisco). China Analysis(68).

Qian, Y. (2000). The Process of China's Market Transition (1978-1998):

The Evolutionary, Historical, and Comparative Perspectives. Journal of Institutional and Theoretical Economics (JITE), 156(1), 151-171.
Reti, P. (2001). China's Path toward a Market Economy: Interview with a Prominent Reformer - Chi Fulin. World Bank Transition Newsletter, Oct/Nov/Dec, 17-19.

Wang, S. (1995). Learning by Debating: The Changing Role of the State in China's Economy and Economics Theories. Policy Studies Journal, 23(1), 11-25.

Xu, Z. (2009). The Unirule Institute of Economics. Zeitschrift für Politikberatung, 2(4), 649-656.

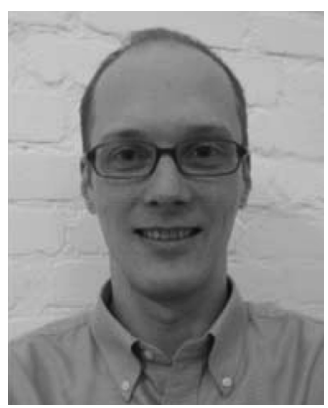

André Lieber ist wissenschaftlicher Mitarbeiter im Büro eines Bundestagsabgeordneten. Von 2004 bis 2006 hat er in Peking gelebt und gearbeitet, zuerst als Gastforscher an der Chinesischen Akademie für Sozialwissenschaften und dann als Berater im Programm Wirtschaftsund Strukturreform der GTZ. Er hat an den Universitäten Mannheim, Nankai (Tianjin/VR China) und Witten/Herdecke Wirtschaftswissenschaften und Chinesisch studiert. EMail: andre.lieber@web.de

\title{
Das Parlament in der medialen Darstellung
}

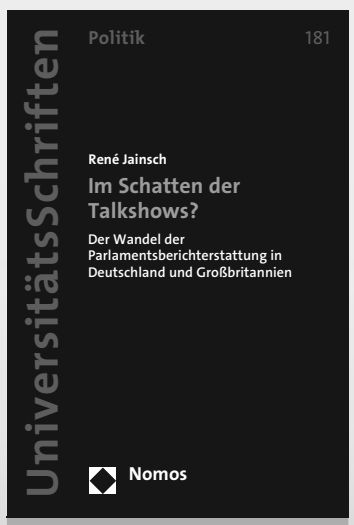

\author{
Im Schatten der Talkshows? \\ Der Wandel der Parlamentsberichterstattung in Deutschland und Großbritannien \\ Von Dr. René Jainsch \\ 2012, 175 S., brosch., 29,- €, ISBN 978-3-8329-6808-3 \\ (Nomos Universitätsschriften - Politik, Bd. 181)
}

Diese wissenschaftliche Studie, die sowohl als internationaler Vergleich als auch als Langzeitvergleich angelegt ist, analysiert Veränderungen in der Berichterstattung über den Deutschen Bundestag und das House of Commons seit den 1980er Jahren. Die Ergebnisse zeigen deutlich, dass das Parlament in der medialen Darstellung von Politik in eine Nebenrolle gerückt ist. 